\title{
On the lattice Hadwiger number of superballs and some other bodies
}

\author{
Serge Vlăduţ*
}

\begin{abstract}
We show that the lattice Hadwiger (=kissing) number of superballs is exponential in the dimension. The same is true for some more general convex bodies.
\end{abstract}

AMS 2010 Classification: 11H31, 05B40, 52C17, 11H71, 14G50;

\section{Introduction}

Let $C$ be a convex body (i.e., a convex closed subset with non-empty intrerior) in a finite-dimensional vector space $\mathbb{R}^{n}$. A Hadwiger family of $C$ is a collection of translates of $C$, all touching $C$ and with pairwise disjoint interiors. The Hadwiger number (or translative kissing number) $H(C)$ of $C$ is the maximum number of translates in a Hadwiger family of $C$. If $B:=\frac{1}{2}(C-C)$ is the central symmetral of $C$ then as was observed already by Minkowski, $\left\{v_{i}+C: i \in I\right\}$ is a Hadwiger family if and only if $\left\{v_{i}+B: i \in I\right\}$ is a Hadwiger family. Also, $\left\{v_{i}+B: i \in I\right\}$ is a Hadwiger family if and only if $\left\{v_{i}, i \in I\right\}$ is a collection of unit vectors in the normed space with $B$ as unit ball, such that $\left\|v_{i}-v_{j}\right\|>1, \forall i \neq j \in I$. We call the Hadwiger number $H\left(B_{X}\right)$ of the unit ball of a finite-dimensional normed space $X$ the Hadwiger number $H(X)$ of $X$. Moreover, in the present note we are concernened only with the lattice Hadwiger number $H_{L}(C)$ defined to be the largest size of a Hadwiger family $\left\{v_{i}+C: i \in I\right\}$ of $C$ that is contained in a lattice packing $\{v+C: v \in \Lambda\}$, where $\Lambda$ is a (full rank) lattice. By Minkowski's observation we can and will suppose that $C=B_{X}$ and consider $H_{L}\left(B_{X}\right)=H_{L}(X)$.

Swinnerton-Dyer [10] showed that $H_{L}(C) \geq n^{2}+n$ for all $n$-dimensional convex bodies $C$ which is the best-known lower bound valid for all convex bodies. Zong [13] (see also [9], Sec. 2.2) conjectured that

$$
H_{L}(C) \geq \Omega\left(c^{n}\right)
$$

for some absolute constant $c>1$ (in fact, in [13 this is formulated rather as a problem than a conjecture). However, until very recently the best asymptotic

\footnotetext{
*Aix Marseille Université, CNRS, Centrale Marseille, I2M UMR 7373, 13453, Marseille, France and IITP RAS, 19 B. Karetnyi, Moscow, Russia, serge.vladuts@univ-amu.fr
} 
lower bound for $H_{L}$ (except the trivial case of hypercubes $B_{\infty}^{n}$ ) was that of the Euclidean ball $B^{n}=B_{2}^{n}$, namely

$$
H_{L}\left(B^{n}\right) \geq 2^{\Omega\left(\log ^{2} n\right)},
$$

attained by the Barnes-Wall lattice, as noted by Leech [7].

The author recently showed in [12] that

$$
H_{L}\left(B^{n}\right) \geq \kappa \cdot 1.015^{n}
$$

for an absolute constant $\kappa>0$ which confirms the conjecture in the case of the Euclidean ball. The purpose of the present note is to show that the same technique can prove the conjecture for many other bodies, for example, classical superballs, i.e. unit balls $B_{p}^{n}$ in the finite dimensional spaces $l_{p}, p \geq 1$, as well as for some of their generalizations considered in the paper [5].

The rest of the note is organized as follows: in Section 2 we recall some necessary results on error-correctig codes, in Section 3 we prove (1.1) for classical superballs by a simplification of the method in 12, and in Section 4 we give further examples of bodies verifying (1.1) for which we need slightly modify the construction.

\section{Preliminaries}

In this section we recall some essential for our constructions definitions and results on linear error-correcting codes.

\section{Error-correcting codes}

Let us recall several facts about (linear error-correcting) codes; for additional information we refer to 8 ; ; see also [11, Ch. 1. We fix a finite field $\mathbb{F}_{q}$.

A $q$-ary linear code is a subspace $C \subseteq \mathbb{F}_{q}^{n}$, where $n$ is called the length of $C, k=\operatorname{dim} C$ being its dimension, the ratio $R=k / n$ is called the rate of $C$. The minimum distance $d=d(C)$ is the minimum Hamming weight $w t(c)$, i.e. the number of nonzero coordinates, of $c \in C \backslash\{0\}$; the ratio $\delta=d / n$ is called the relative minimum distance. We say in this case that $C$ is an $[n, k, d]_{q}$-code.

\section{Algebraic geometry codes}

All our curves here and below are smooth projective absolutely irreducible over a finite field $\mathbb{F}_{q}$; let $Y$ be such a curve of genus $g$, let $D$ be an $\mathbb{F}_{q}$-rational divisor of degree $a \geq g-1$, and let (see, e.g., [11, Sec.2.2)

$$
L(D)=\left\{f \in \mathbb{F}_{q}(Y):(f)+D \geq 0\right\}
$$

be the associated function space. For a set $\mathcal{P}=\left\{P_{1}, \ldots, P_{n}\right\}$ of $\mathbb{F}_{q}$-rational points on $Y$ with $\mathcal{P} \cap \operatorname{Supp} D=\emptyset$ the evaluation map

$$
e v_{\mathcal{P}}: L(D) \longrightarrow \mathbb{F}_{q}^{n}, e v_{\mathcal{P}}(f)=\left(f\left(P_{1}\right), \ldots, f\left(P_{n}\right)\right)
$$


is well defined. Whenever $a<n$ this map is injective and its image is a linear $q$-ary code $C(Y, D, \mathcal{P})$ of length $n$, dimension $k \geq a-g+1$ (by the RiemannRoch theorem), and distance $d \geq n-a$ (since the number of zeros of a function cannot exceed the number of poles). In fact, one can dispense with the above condition $\mathcal{P} \bigcap \operatorname{Supp} D=\emptyset$ not spoiling the parameters of the codes $C(Y, D, \mathcal{P})$.

Algebraic geometry codes (AG-codes below) have good parameters when the ratio of the number of $\mathbb{F}_{q}$-rational points on the curve to its genus is high enough; an optimal family of such curves is contained, e.g., in [6].

\section{Codes with many light vectors}

Recall the following result from [1] :

Theorem 2.1 Let $q=2^{2 s}, s=3,4, \ldots$ be fixed. Then for any $\delta_{1}<\delta<\delta_{2}$ there exists a sequence of binary linear codes $\left\{C_{n}\right\}$ of length $n=q N, N \longrightarrow \infty$ and distance $d_{n}=n \delta / 2$ such that

$$
\frac{\log A_{d_{n}}}{n} \geq \frac{E_{s}(\delta)}{2^{2 s}}-o(1)
$$

Here $A_{d_{n}}$ is the number of minimum weight vectors in $C_{n}$,

$$
E_{s}(\delta)=H(\delta)-\frac{2 s}{2^{s}-1}-\log \frac{2^{2 s}}{2^{2 s}-1},
$$

and $H(\delta)=-\delta \log \delta-(1-\delta) \log (1-\delta)$ is the binary entropy. Here and below all logarithms are binary. The function $E_{s}(\delta)$ has two zeros $0<\delta_{1}<\delta_{2}<1-2^{-2 s}$ and is positive for $\delta_{1}<\delta<\delta_{2}$. A simple calculus shows that the function

$$
E(\delta):=\max _{s \geq 3}\left\{2^{-2 s} E_{s}(\delta)\right\}
$$

is everywhere strictly positive on the whole $] 0,1[$, strictly increasing on $\left.] 0, \frac{1}{2}\right]$, strictly decreasing on $\left[\frac{1}{2}, 1[\right.$, and its maximum $M$ equals

$$
M=E(0.5)=E_{3}(0.5) / 64=\left(\frac{1}{7}-\log \frac{64}{63}\right) / 64 \approx 0.001877 \ldots .
$$

Theorem 2.1 is a simple consequence of a result concerning AG-codes which uses an averaging procedure applied to the set of linearly equivalent classes of $\mathbb{F}_{q}$-rational positive divisors $D$ with $\operatorname{deg}(D)=a$ which form the set $J_{Y}\left(\mathbb{F}_{q}\right)$ of $\mathbb{F}_{q}$-rational points on the Jacobian $J_{Y}$ of a curve $Y$ from an optimal family.

\section{The case of superballs}

We beging with a consruction which is an extremely simplified and slightly modified version of constructions from [2], 3] (see also Chapter 8 in [4] ), which permit to construct good lattices from good codes. 


\section{Simplified construction D.}

Let $C$ be a linear binary codes with parameters $[n, k, d]$; we can and will consider $C$ as a subset of $\mathbb{R}^{n}$. Fix an integer $t \geq 2$. Let $C_{d}=c_{1}, \ldots, c_{a}$ be the set of minimum weight vectors in $C$, where $a=A_{d}(C)$. We define $\Lambda$ as the lattice in $\mathbb{R}^{n}$ generated by $\mathbb{Z}^{n}$ and the vectors $\left\{c_{i} / t\right\}$ for $i=1, \ldots, a$. We express this in the following way:

$$
\Lambda=\Lambda(C, t)=\mathbb{Z}^{n}+\lambda\left\langle C_{d}\right\rangle, \lambda=1 / t
$$

Note that any $c \in \Lambda \backslash\{0\}$ can be written as follows:

$$
v=\frac{r}{t} v_{0}+v_{1}, v_{0}, v_{1} \in \mathbb{Z}^{n}, w t\left(v_{0}\right) \geq d, r \in\{0,1\} .
$$

Our aim is to formulate some sufficient conditions on $C, t$ and $X$ under which the lattice $\Lambda(C, t)$ gives an estimate of the form (1.1) for $B_{X}$ corresponding to the norm $\|\cdot\|_{X}$ on $\mathbb{R}^{n}$. It means that we want minorate the number $N_{s}(\Lambda, X)$ of the shortest nonzero vectors in $\Lambda(C, t)$ exponentially in $n$. We look for conditions under which the set of the shortest nonzero vectors contains $\lambda C_{d}$. Since $N_{s}(\Lambda, X)$ is invariant under a rescaling $\Lambda \rightarrow \nu \Lambda$, we can always suppose that the minimum norm is one, applying a rescaling, if necessary. For classical superballs we get

Theorem 3.1 Let $p \geq 1$ and $X=l_{p}^{n}$. Then $B_{X}$ verifies (1.1) with

$$
c=2^{M}=\left(63 \cdot 2^{-41 / 7}\right)^{1 / 64}>1.0013 .
$$

Proof. We prove this first for $p=1$, and then for any $p \geq 1$.

If $s=3, q=64, \delta=1 / 2, n=q N=4 d$, and $C$ is an $[n, k, d]$-code from Theorem 2.1 (and thus $n=64 N, k=\lceil 15 N / 7\rceil, d=16 N$ with $N \rightarrow \infty$ ) then for $X=l_{1}^{n}, t=d, \lambda=1 / d, B=B_{1}^{n}$ we have

$$
H_{L}\left(B_{1}^{n}\right) \geq N_{s}\left(\Lambda, l_{1}\right) \geq h \cdot 2^{M n}=h\left(63 \cdot 2^{-41 / 7}\right)^{n / 64}>h(1.0013)^{n}
$$

for an absolute constant $h>0$ which confirms the conjecture (1.1) in this case. Indeed, the minimum norm $(=1)$ vectors in $\Lambda(C, t)$ are either the standard basis vectors $e_{i}, i=1, \ldots, n$ or the vectors from $\lambda C_{d}$. This follows from (3.2) since if $r \neq 0$ then $v \in \frac{1}{t} \mathbb{Z}^{n}, w t(v) \geq d$ and thus $\|v\|_{1} \geq d / d=1$, and if $r=0$ then $v_{1} \neq 0$ and $\|v\|_{1}=\left\|v_{1}\right\|_{1} \geq 1$.

More generally, for the same $C$ and $X=l_{p}^{n}, p \geq 1, t=\left\lceil d^{\frac{1}{p}}\right\rceil, \lambda=1 / t, B=B_{p}^{n}$ we again get

$$
H_{L}\left(B_{p}^{n}\right) \geq N_{s}\left(\Lambda, l_{p}\right) \geq h \cdot 2^{M n}>h(1.0013)^{n}
$$

for an absolute positive constant $h$ which confirms the conjecture (1.1) for the classical superballs, i.e. unit balls $B_{p}^{n}$ in $l_{p}, p \geq 1$. Indeed, any vector in $\lambda C_{d}$ has the norm $\nu:=d^{\frac{1}{p}} \lambda=d^{\frac{1}{p}} /\left\lceil d^{\frac{1}{p}}\right\rceil \leq 1$ which is the minimum nonzero norm of elements in $\Lambda(C, t)$ by (3.2), which gives the result after the rescaling $\Lambda \rightarrow \nu^{-1} \Lambda$. 
Remark 3.2. For $p=2$ this result essentially coinsides with the result of the simplest construction in [12. However, other results of [12] are considerably better, that is, have larger constant $c$ in (1.1). The reason is that they are obtained applying rather advanced construction E from [2], 3] which is adapted for Euclidean balls and has no clear counterpart for $p \neq 2$.

\section{Some generalizations}

The authors of [5] consider the following more general superballs, given by the inequality

$$
\left\{f\left(x_{1}, \ldots, x_{k}\right)^{p}+f\left(x_{k+1}, \ldots, x_{2 k}\right)^{p}+\ldots+f\left(x_{n-k+1}, \ldots, x_{n}\right)^{p} \leq 1\right\}
$$

on $x=\left(x_{1}, \ldots, x_{k}, x_{k+1}, \ldots, x_{2 k}, \ldots, x_{n-k+1}, \ldots, x_{n}\right) \in \mathbb{R}^{n}$, where $f$ is some $k$-dimensional body's distance function, and $n$ is a multiple of $k$. Moreover, they consider certain inhomogeneous generalizations of (4.1).

Let us show that (1.1) remains valid in that inhomogeneous setting. Let us fix positive integers $k, l, m \geq 1$, a finite collection $K=\left\{k_{1}, k_{2}, \ldots, k_{m}\right\}$ of positive integers with $\max \left\{k_{j}: j \in[1, m]\right\}=k$, a finite collection of exponents $P=\left\{p_{1}, p_{2}, \ldots, p_{l}\right\} \subset[1, \infty)$ and a finite collection $F=\left\{f_{1}, f_{2}, \ldots, f_{m}\right\}$, where $f_{i}, i=1, \ldots, m$ is a Minkowski distance function on $\mathbb{R}^{k_{i}}$ for $k_{i} \in K$. We suppose for simplicity that each function $f_{i}, i=1, \ldots, m$ verifies the following, relatively moderate, monotonicity condition:

$$
\forall a_{2}, \ldots, a_{k_{i}} \in \mathbb{R}, f_{i}(1,0, \ldots, 0) \leq f_{i}\left(1, a_{2}, \ldots, a_{k_{i}}\right)
$$

which is true, e.g., for a separable $f_{i}\left(x_{1}, \ldots, x_{k_{i}}\right)=f_{1}\left(x_{1}\right)+f_{2}\left(x_{2}, \ldots, x_{k_{i}}\right)$; note, however, that this condition can be considerably weakened (if not eliminated completely). Define then a family $\mathcal{B}=\left\{B_{X}^{(n)}\right\}, B_{X}^{(n)} \subset \mathbb{R}^{n}, n \geq n_{0}$ of generalized superballs by the inequalities

$$
B_{X}^{(n)}=\left\{\sum_{j=1}^{t(n)}\left(f_{j}\left(\mathbf{x}_{j}\right)\right)^{p_{j}} \leq 1\right\},
$$

where $f_{j} \in F, p_{j} \in P$ and $\mathbf{x}_{j}=\left(x_{i_{1}}, \ldots, x_{i_{k(j)}}\right)$, i.e. $f_{j}$ depends of $k(j) \in K$ variables in $S_{j}$ for some partition $\left\{S_{j}\right\}, j=1, \ldots, t(n)$ of $[1, n]$ such that

$$
\left|S_{j}\right|=k(j) \in K, \sum_{j=1}^{t(n)} k(j)=n .
$$

We denote by $X^{(n)}$ the Minkowski space with the unit ball $B_{X}^{(n)}$.

Here is a small example of such body

$B_{X}^{(7)}=\left\{x \in \mathbb{R}^{7}: 2 \sqrt{x_{1}^{4}+5 x_{7}^{4}}+\left(x_{2}^{2}+x_{1} x_{2}+3 x_{3}^{2}\right)^{\frac{3}{4}}+\left(2\left|x_{4}\right|^{\frac{5}{4}}+\max \left\{\left|x_{5}\right|,\left|x_{6}\right|\right\}^{\frac{5}{4}}\right)^{\frac{7}{3}} \leq 1\right\}$.

In this notation we have 
Theorem 4.1 Under the above conditions $B_{X}^{(n)} \in \mathcal{B}$ verifies (1.1) with

$$
c=2^{M \mu}>1
$$

where $\mu=\mu(k, l, m)=\frac{1}{k l m}$.

Proof.

Let us take $q=64, s=3, \delta=\frac{1}{2}$ and an $\left[n^{\prime}=4 d, k^{\prime}, d\right]$-code $C^{\prime}$ from Theorem 2.1 with and put $n=n^{\prime} / \mu=n^{\prime} k l m=4 k l m d$. We are going to complete (to lengthen) $C^{\prime}$ by zeros to get an $\left[n, k^{\prime}, d\right]$-code $C$ and then apply (3.1). To do that we consider first the family $L=\left\{f_{j}, j=1, \ldots, t(n)\right\}$. Since $f_{j} \in F$ and $|F|=m$, there exists $j_{0} \in[1, m]$ s.t. $\left|T_{0}\right| \geq n / m=k l n^{\prime}$ for $T_{0}=\left\{j: f_{j}=f_{j_{0}}\right\}$, and we fix such $T_{0}$. Further we consider the family of exponents $\left\{p_{j}, j \in T_{0}\right\}$. Since any $p_{j} \in P$ and $|P|=l$, we get as before that $\left|T_{1}\right| \geq k l n^{\prime} / l=k n^{\prime}$ for some $j_{1} \in[1, l]$ and $T_{1}=\left\{j \in T_{0}: p_{j}=p_{j_{1}}\right\}$. Now we define an embedding $\Phi: \mathbb{F}_{2}^{n^{\prime}} \longrightarrow \mathbb{F}_{2}^{n}$ sending the components of a vector $v \in \mathbb{F}_{2}^{n^{\prime}}$ one by one to the first component of $\mathbf{x}_{j}$ for $j \in T_{1}$ setting all other components to zero. Finally, put $C=\Phi\left(C^{\prime}\right)$. One easily calculates then that

$$
\forall v \in C_{d},\|v\|_{X^{(n)}}=f_{j_{0}}(1,0, \ldots, 0)^{p_{j_{1}}} d=: \nu_{n} .
$$

Define

$$
\rho:=\min \left\{f_{j_{0}}(v): v \in \mathbb{Z}^{k_{j_{0}}} \backslash\{0\}\right\}
$$

and let $v \in \Lambda(C, t) \backslash\{0\}$ for an integer $t \geq 2$. We write $v=\frac{r}{t} v_{0}+v_{1}$ as in (3.2) and consider two cases: $r \neq 0$ and $r=0$. For $r \neq 0$ the condition (4.2) implies that

$$
\|v\|_{X^{(n)}} \geq f_{j_{0}}\left(\frac{1}{t}, 0, \ldots, 0\right)^{p_{j_{1}}} \cdot d=\nu_{n} / t^{p_{j_{1}}}
$$

whereas for $r=0$ we get $\|v\|_{X^{(n)}} \geq \rho^{p_{j_{1}}}$ by the definition of $\rho$. We take then $t=\max \left\{\left\lceil\nu_{n}\right\rceil,\left\lceil\rho^{-1}\right\rceil\right\}, \lambda=1 / t$ in (3.1). The above inequalities for $\|v\|_{X^{(n)}}$ show that elements of $\frac{1}{t} C_{d}$ are of minimum nonzero norm $\nu_{n} / t^{p_{j_{1}}}$ and thus

$$
N_{s}\left(\Lambda, X^{(n)}\right) \geq A_{d}(C)=2^{M n^{\prime}}=2^{M \mu n}
$$

which finishes the proof after an appropriate rescaling.

Remark 4.2. It is clear that the above conditions can be generalized further at the expense of more cumbersome formulations and further deterioration of constants in (1.1). Moreover, there are some evident classes of norms, e.g. those invariant under coordinate permutations, for which it is natural to seek candidates for validity of (1.1), but we do not explore here this possibility. However, Theorems 3.1 and 4.1 give already an argument in support of Conjecture (1.1).

Remark 4.3. We do dot care here about the density of our packings; note, however, that the constructed families are "asymptotically good" (i.e. having the packing density $\Omega\left(2^{-\kappa n}\right)$ for a constant $\left.\kappa>0\right)$ albeit very poor for their density ( $\kappa$ is very large). 


\section{References}

[1] A. Ashikhmin, A. Barg, S. Vlăduţ, Linear codes with exponentially many light vectors. J. Combin. Theory Ser. A 96 (2001), 396-399.

[2] A. Bos, J.H. Conway, N.J.A. Sloane, Further lattice packings in high dimensions. Mathematika 29 (1982), 171-180.

[3] E.S. Barnes, N.J.A. Sloane, New lattice packings of spheres. Canad. J. Math. 35 (1983), 117-130.

[4] J.H. Conway, N.J.A. Sloane, Sphere packings, lattices and groups. With contributions by E. Bannai, J. Leech, S. P. Norton, A. M. Odlyzko, R. A. Parker, L. Queen and B. B. Venkov. Springer-Verlag, NY, 1988. xxviii+663 pp.

[5] N.D. Elkies, A.M. Odlyzko, J.A. Rush, On the packing densities of superballs and other bodies, Invent. math. 105 (1991), 613-639.

[6] A. Garcia, H. Stichtenoth, A tower of Artin-Schreier extensions of function fields attaining the Drinfeld-Vlăduţ bound, Invent. Math. 121 (1995), 211222 .

[7] J. Leech, Some sphere packings in higher space, Canad. J. Math. 16 (1964), $657-682$.

[8] F.J. MacWilliams, N.J.A. Sloane, The theory of error-correcting codes, NH, Amsterdam, 1981.

[9] K.J. Swanepoel, Combinatorial distance geometry in normed spaces, New Trends in Intuitive Geometry, Springer, 2018, 407-458.

[10] H.P.F. Swinnerton-Dyer, Extremal lattices of convex bodies, Proc. Cambridge Philos. Soc. 49 (1953),161-162.

[11] M. Tsfasman, S. Vlăduț, D. Nogin, Algebraic geometric codes: basic notions. Math. Surv. Monogr., 139. AMS, Providence, RI, 2007. xx+338pp.

[12] S. Vlădut, Lattices with exponentially large kissing numbers, Moscow Journal of Combinatorics and Number Theory, 8 (2019), no 2 (arXiv:1802.00886).

[13] C. Zong, The kissing number, blocking number and covering number of a convex body, Surveys on discrete and computational geometry, Contemp. Math., vol. 453, Amer. Math. Soc., Providence, RI, 2008, 529-548. 